\title{
El Estudio del Proceso del Cambio según el Modelo de Asimilación: Una Introducción Teórica y Metodológica
}

\section{The Study of the Process of Change Following the Assimilation Model: A Theoretical and Methodological Introduction}

\author{
Isabel Caro Gabalda \\ Universidad de Valencia \\ Irene Rojo Serrano \\ Práctica privada
}

\author{
Anna Allepuz Faus \\ Práctica privada \\ Ainara Yera Sabater \\ Asociación Valenciana de Esclerosis Lateral Amiotrófica
}

\begin{abstract}
Resumen. Con este trabajo se busca la descripción teórica y metodológica del modelo de asimilación y de algunos de los principales resultados. El modelo de asimilación se ha desarrollado como un modelo de procesos integrador que permite describir el proceso de cambio en cualquier enfoque de terapia. Este trabajo describe el modelo y cómo debe ser el procedimiento a seguir cara al desarrollo de estudios de asimilación. Define, además, sus conceptos principales, como asimilación, voces dominantes y no dominantes y puente de significado y se presenta la Escala de Asimilación de Experiencias Problemáticas (APES). Se resumen algunos de los principales resultados alcanzados, como por ejemplo, la relación entre asimilación y cambio terapéutico, la relación entre no asimilación y ausencia de cambio terapéutico. Se hace mención, igualmente, al patrón característico de asimilación encontrado en terapias de corte cognitivo, es decir, un patrón de tipo dentado.
\end{abstract}

Palabras clave: APES, cambio terapéutico, investigación de procesos, modelo de asimilación.

\begin{abstract}
The main aim of this paper is the theoretical and methodological description of the assimilation model. The assimilation model has been developed as an integrative process research model that allows us to describe the process of change in any therapeutic approach. This paper describes the model and how should be its procedure. Besides, the paper defines its main concepts, such as assimilation, dominant and nondominant voices, meaning bridge and describes The Assimilation of Problematic Experiences Scale (APES). Some of the main results are shown, such as the one that relates change with assimilation and no assimilation and no change. Also, it is described the characteristic sawtoothed pattern of assimilation found in cognitive therapies.
\end{abstract}

Keywords: APES, assimilation model, process research, therapeutic change.

El presente artículo se centra en el modelo de asimilación de experiencias problemáticas. Este modelo busca describir el proceso del cambio terapéutico, fundamentalmente desde una perspectiva cualitati-

La correspondencia sobre este artículo debe enviarse a la primera autora a Facultad de Psicología. Universidad de Valencia. Avda. Blasco Ibañez, 21.46010 Valencia. E-mail: Isabel.Caro@uv.es va. El modelo se origina en los trabajos de Stiles y sus colaboradores (Stiles et al., 1990, 1991) que, dentro de esta perspectiva de procesos, pretendían desarrollar un enfoque transteórico e integrador. Es decir, mediante el modelo de asimilación podemos estudiar el cambio terapéutico en cualquier perspectiva terapéutica. En este sentido presentaremos el 
modelo de asimilación, así como algunos de los principales resultados encontrados por nuestro grupo de investigación.

\section{El modelo de asimilación de experiencias problemáticas: Introducción}

Según Stiles (2006) el modelo de asimilación representa una teoría sobre el cambio psicológico que pretende ofrecer una alternativa conceptual y metodológica a los estudios tradicionales que fracasaban en su vinculación del proceso terapéutico con el resultado usando modelos estadísticos lineales (Stiles \& Shapiro, 1989, 1994). Mientras que el proceso terapéutico se refiere a acontecimientos en sesión medidos en una escala temporal de segundos o minutos, el resultado terapéutico se ha medido en una escala de meses o de años. Lo que plantea el modelo de asimilación es ofrecer una alternativa a todo ello que supere la estadísticamente improbable vinculación entre efectos que ocurren en un minuto con otros que ocurren a lo largo de varios (o muchos meses). ¿Qué plantea, pues, el modelo de asimilación para lograr abordar desde esta perspectiva el proceso del cambio?

El modelo de asimilación, (Stiles et al., 1990, 1991) describe el proceso por el que el paciente pasa, en terapia, cuando logra asimilar, en su sí mismo, aquellas experiencias problemáticas o dolorosas con las que acude a terapia o que surgen en el marco del trabajo conjunto paciente-terapeuta.

El modelo integra cuestiones importantes de diversos modelos. Así, tiene en cuenta factores $\operatorname{cog}$ nitivos (por ejemplo, en los niveles de Clarificación y de Insight), emocionales (por ejemplo, en los niveles de Surgimiento de la experiencia problemática) y conductuales (por ejemplo, cuando el paciente pone en marcha cursos de acción diferentes para resolver su experiencia problemática, en los niveles 5 o de Aplicación). Al integrar estas variables, nos facilita identificar procesos comunes de cambio y articular diferencias sistemáticas entre distintos enfoques terapéuticos.

En este sentido, el modelo se ha estudiado tanto en contextos terapéuticos (Caro, 2006a,b, 2007, Detert, Llewelyn, Hardy, Barkham, \& Stiles, 2006; Good- ridge \& Hardy, 2009; Honos-Webb, Surko, Stiles, \& Greenberg, 1999), como no terapéuticos (Henry, Stiles, \& Biran, 2005). Fundamentalmente, los estudios se han hecho con pacientes depresivos (Brinegar, Salvi, Stiles, \& Greenberg, 2006; Osatuke $\&$ Stiles, en prensa), con problemas de ansiedad (Caro Gabalda, 2006a, 2007, 2008), o con trastorno de estrés postraumático (Osatuke \& Stiles, en prensa) (véase, Stiles, 2002, para una revisión de los principales estudios realizados). Pero también se han hecho estudios con pacientes que presentan una sintomatología más grave como trastornos disociativos (Humphreys, Rubin, Knudson, \& Stiles, 2005) y trastornos de personalidad (Osatuke \& Stiles, 2006).

La mayoría de los estudios con la APES que acabamos de reseñar se han centrado en el proceso de asimilación de los pacientes sin incluir el papel del terapeuta. Estudios recientes se preocupan de integrar al terapeuta en estos análisis como un elemento clave, facilitador del cambio, entendido como progreso en la asimilación (véase, por ejemplo, Goldsmith, Mosher, Stiles, \& Greenberg, 2008).

Desde un punto de vista clínico el modelo nos puede informar del momento concreto en el que se encuentra el paciente y guiar las subsecuentes fases de intervención (Stiles, Shapiro, Harper, \& Morrison, 1995). No obstante, el modelo no es un modelo prescriptivo, es decir, no nos dice cómo debemos de-sarrollar la terapia, o qué debemos hacer para producir un cambio, por lo que puede utilizarse en diversos marcos terapéuticos, ya que no nos "fuerza" a seguir determinados pasos o realizar determinadas actividades. Esto es, el modelo se aplica "a posteriori" para describir el proceso del cambio en terapias realizadas sobre la base de sus propios requisitos, metas, técnicas, etc. Por todo lo cual es un modelo que tiene un gran prestigio dentro del campo de la investigación de procesos y que incluso ha sido considerado como un modelo "prometedor y posiblemente efectivo" en el marco de las relaciones terapéuticas empíricamente validadas (Castonguay \& Beutler, 2006; Norcross, 2002).

A niveles teóricos, el modelo parte, ante todo, del trabajo de Piaget sobre esquemas, del de Rogers sobre el desarrollo del sí mismo y del modelo de Elliott que definió la secuencia sistemática de cambios mediante la cual podíamos representar una 
experiencia problemática durante la psicoterapia. Teóricamente, una experiencia es problemática porque nos desequilibra emocionalmente. Estas experiencias ocurren repetidamente como respuesta a una variedad de acontecimientos (interpersonales, normalmente), pero son incompatibles con las formas usuales de pensar y actuar del individuo y no se pueden representar de forma correcta en la conciencia. En su lugar, las experiencias problemáticas se niegan, se distorsionan o se representan de forma equivocada, produciendo sentimientos disfóricos o conducta desadaptativa (Stiles, Meshot, Anderson, \& Sloan, 1992) (véase, más adelante, apartado de conceptos principales).

Siguiendo todo esto, el grupo de Stiles ha desarrollado la Escala de Asimilación de Experiencias Problemáticas (Assimilation of Problematic Experiences Scale) o APES, que consta de 8 niveles o fases.

\section{La Escala de Asimilación de Experiencias Problemáticas o APES}

La escala que inicialmente se llamaba Assimilation of Ideas Scale (o AIDS; Stiles, Morrison, \& Haw, 1987) pasó a llamarse Escala de Asimilación de Experiencias Problemáticas en su primera publicación (Stiles et al., 1991). En sus diversos trabajos se han propuesto 8 niveles principales en el proceso de asimilación que forman la propia escala APES (Stiles et al., 1991; HonosWebb \& Stiles, 1998). Estos nos aparecen en el cuadro 1. La Escala es independiente del contenido problemático, la población diagnóstica o el enfoque terapéutico.

Queremos hacer constar que la escala permite niveles intermedios como un intento de ofrecer una estimación más precisa del nivel APES en el que se encuentra el paciente y que los niveles APES se pue-

Cuadro 1. Niveles en el proceso de asimilación (a partir de Stiles, Honos-Webb \& Lani, 1999)

\section{NIVEL O. SUPRESIÓN/DISOCIACIÓN:}

El contenido no está formado; el paciente no se da cuenta del problema. El paciente no trabaja en el problema.La experiencia se considera evitada o desviada si hay evidencia de evitar activamente temas emocionalmente molestos (i.e., cambiar de tema).

\section{NIVEL 1. PENSAMIENTOS NO DESEADOS/ EVITACIÓN:}

Control/evitación. El contenido refleja el surgimiento de pensamientos asociados con el malestar. El paciente prefiere no pensar sobre ello; los temas los saca el terapeuta o las circunstancias externas. El terapeuta pregunta algo y el paciente cambia de tema, algo así como "tengo que ser duro y no pensar en esto". El afecto es más destacado que el contenido e implica fuertes sentimientos negativos -ansiedad, miedo, cólera, tristeza.

\section{NIVEL 2. RECONOCIMIENTO VAGO/SURGIMIENTO:}

El paciente se da cuenta de la existencia de una experiencia problemática, y describe pensamientos poco agradables asociados con los pensamientos, pero no puede formular el problema con claridad. Sabe lo que le molesta. El afecto incluye dolor psicológico agudo o pánico asociados con los pensamientos problemáticos y las experiencias.

\section{NIVEL 3. ENUNCIAR EL PROBLEMA/CLARIFICACIÓN:}

Ves lo que pasa y lo que lo impide. El contenido incluye un enunciado claro del problema -algo en lo que se puede trabajar. El paciente enuncia el problema pero no hace ninguna conexión sobre la causa del problema. El afecto es negativo pero manejable, no hay pánico.

\section{NIVEL 4. COMPRENSIÓN/INSIGHT:}

La experiencia problemática se coloca en un esquema, es formulada y comprendida con claros vínculos conectivos. El afecto puede ser variado, con algunos reconocimientos desagradables, pero con curiosidad o incluso con sorpresa del tipo del "ajá". Niveles del 4.1 al 4.9 reflejan progresivamente mayor claridad o generalización de la comprensión, asociada, por lo general, con un afecto positivo (o negativo disminuyendo).

\section{NIVEL 5. APLICACIÓN / ELABORACIÓN:}

La comprensión se emplea para trabajar en el problema; hay una referencia a esfuerzos específicos para resolver el problema, aunque sin un éxito completo. El paciente puede describir que está considerando alternativas o seleccionando, sistemáticamente, cursos de acción.

\section{NIVEL 6. SOLUCIÓN DEL PROBLEMA:}

El paciente logra una solución a un problema específico. El afecto es positivo, satisfecho, orgulloso del logro. Niveles del 6.1 al 6.9 reflejan generalizar la solución a otros problemas y construir las soluciones sobre patrones usuales o habituales de conducta. El paciente ha practicado la solución fuera de la consulta.

NIVEL 7. DOMINIO: El paciente emplea, con éxito, soluciones en nuevas situaciones; esta generalización es bastante automática, no destacada. El afecto es positivo cuando se habla del tema, pero neutro (i.e. esto no es ya un problema o algo por lo que preocuparse). El problema ha dejado de ser un problema. 
den comunicar a través de números. Así, un código de 2.5 indicaría que el paciente (en cuanto al párrafo codificado) se encuentra a medio camino entre Surgimiento (2) y Clarificación (3). Diversos estudios han apoyado dicha cuestión (Brinegar, Salvi, Stiles, \& Greenberg, 2006; Reid \& Osatuke, 2006). El empleo de números se justifica para comunicar mejor a la audiencia los niveles APES (Osatuke \& Stiles, en prensa; Stiles, 2006).

\section{Principales estudios con la APES}

A niveles de investigación, la APES se encuadra dentro de la investigación de procesos y en concreto en el denominado paradigma de acontecimientos (Rice \& Greenberg, 1984) que estudia episodios breves que comparten un marcador común. Los trabajos con la APES extienden este paradigma al examinar problemas seleccionados y cómo se trabaja una y otra vez con ellos a lo largo de muchas sesiones (Stiles, 2003; Stiles, Barkham, Shapiro, \& FirthCozens, 1992).

La APES trabaja, pues, con patrones terapéuticos (en una escala pequeña, microanalítica), que se estudian intensivamente y en un contexto. Aunque la escala permite estudios cuantitativos (Field, Barkham, Shapiro, \& Stiles, 1994) la mayor parte de sus trabajos se ha fundamentado en una metodología cualitativa. Y de hecho el modelo y la escala se presentan como una metodología cualitativa.

Además, la mayoría de los estudios realizados para "validar" la APES y el modelo de asimilación ha seguido la perspectiva que se conoce como estudio de caso para construir una teoría. Es decir, la APES está en un desarrollo continuo a medida que los investigadores logran comprender aspectos nuevos del progreso terapéutico, medido con la APES en distintos contextos y muestras (Stiles, 2003).

En sus trabajos el grupo de investigación ha obtenido diversos resultados que ofrecemos, a continuación, de forma resumida (Caro, 2006a,b,2007, 2008; Caro \& Stiles, 2009; Detert et al., 2006; Brinegar et al., 2006; Stiles et al., 1990, 1991, Stiles, Meshot et al., 1992, Stiles, Barkham, et al., 1992; Honos-Webb et al., 1999; Osatuke et al., 2004, 2005).

Relaciones entre asimilación y cambio. Una de las conclusiones principales, por ser ésta la finalidad del desarrollo del modelo, se refiere a aquella que permite establecer una relación entre cambio terapéutico y progreso en la asimilación e, igual pero al contrario, es decir, entre ausencia de cambio terapéutico y dificultades en el proceso y progreso en la asimilación. Es decir, pacientes con éxito terapéutico se mueven en niveles elevados de la escala APES, mientras que pacientes con poco éxito se mueven en niveles bajos de la escala APES.

Diferencias entre terapias en función del modelo de asimilación. Las distintas psicoterapias se focalizan en rangos distintos a lo largo del continuo de asimilación y movilizan estrategias diferentes en función del grado de asimilación de las experiencias del paciente. Así, las terapias experienciales y psicodinámicas se focalizan en experiencias no asimiladas y trabajan hacia su comprensión. Los resultados encontrados señalan que estas terapias aparecen en un rango APES que va desde los niveles bajos, como 1, Pensamientos no deseados o 2, Surgimiento, hasta los intermedios, por ejemplo el nivel 4 o Insight. Por su parte, se asume que las terapias cognitivas y las conductuales se focalizan en problemas conocidos y trabajan en la aplicación de soluciones racionales en situaciones prácticas. Así, se mueven en un rango APES desde los niveles intermedios, como el 3, Clarificación y 4 Insight, hasta los altos, como 5 (Aplicación) y 6 (Solución del Problema). Aunque ésta no es una distinción absoluta.

Patrón de asimilación. Quizás uno de los resultado más notables, ha sido que la hipótesis del continuo APES, es decir, de la secuencia sistemática de cambios asumida inicialmente no se ha comprobado. Es decir, aunque el progreso en terapia se relaciona con el paso de un nivel de la escala a otro y con la asimilación final, este paso puede tener momentos en los que se vuelve atrás en la escala e incluso producirse saltos entre niveles. Siendo estos retrocesos muy marcados en terapias de corte cognitivo y cognitivo-conductual y no tan destacados en terapias de corte experiencial en el que el paso entre niveles es menos dentado, siendo más fluido.

Se han ofrecido diversas respuestas al por qué de los retrocesos, aunque básicamente los datos obtenidos señalan a dos principales: el concepto (tomado de Vygotski) de la zona de desarrollo próximo y la 
hipótesis de la balanza, que desde un marco cognitivo describe el trabajo conjunto paciente-terapeuta en una terapia cognitiva como la terapia lingüística de evaluación que es sobre la que se han hecho la mayoría de nuestros estudios.

\section{Conceptos principales del modelo APES}

Antes de exponer cada uno de los niveles de la escala APES conviene que definamos algunos términos. Lo primero que debe quedar claro es que una experiencia problemática será cualquier percepción, intención, impulso, actitud, deseo, fantasía, o idea que causa malestar psicológico cuando aparece en la conciencia o se pone en acción (Stiles, et al., 1990).

Otro concepto fundamental es el de voz. Aunque el modelo surgió asociado al concepto de esquema, con el tiempo, el modelo empezó a utilizar el concepto de voz en lugar del de esquema.

El modelo de Stiles plantea una propuesta de sí mismo (Honos-Webb \& Stiles, 1998; Stiles, HonosWebb, \& Lani, 1999; Honos-Webb, et al., 1999; Stiles, 2002) cercana a la de Hermans (Hermans, Kempen, \& van Loon, 1992; Hermans, 1996), con su concepto de voz. El motivo por el que el grupo de Stiles cambió hacia el concepto de voz (HonosWebb \& Stiles, 1998), se explica por considerar a éste como una metáfora útil que destaca el papel activo de los rastros de todas nuestras experiencias. Para ellos, una voz sería una metáfora para conceptos que se utilizan dentro de cada modelo, por ejemplo, el concepto de pensamiento automático en psicoterapias cognitivas. No obstante, el concepto de esquema se mantiene dentro del concepto de puente de significado (véase éste más adelante).

Desde la nueva formulación (la más utilizada en la actualidad en los diversos estudios) podemos hablar de voces dominantes y de voces no dominantes. Las voces dominantes están compuestas por nuestros recursos, nuestra forma habitual de pensar, sentir y comportarnos. Es decir, todos nuestros supuestos, creencias, evaluaciones, formas de afrontar las cosas, las experiencias asimiladas, etc. que utilizamos para dar sentido, guiar nuestra conducta, saber qué debemos hacer cuando tenemos una nueva experiencia, que si no es asimilada se convierte en una experiencia problemática, que es lo que se denomina voces no dominantes.

Nuestras experiencias son, entonces, problemáticas porque tenemos creencias, pensamientos, evaluaciones opuestas que las hacen problemáticas. Cualquier experiencia que tengamos será sólo una experiencia no experimentada como problemática, si no surge alguna voz que la contradiga y la haga problemática. Por ejemplo, si un paciente tiene como voz dominante tener éxito en todo lo que emprende, y no lo consigue ya que tiene un notorio fracaso (voz no dominante), a menos que asimile esta experiencia, va a tener problemas. Como ya se ha dicho, la asimilación va a ser consecuencia de un puente de significado.

¿Qué significado tiene asimilar? Originalmente, asimilar suponía que cuando aparece una experiencia nueva, un "esquema" la acoge -la integra, la explica, y la incorpora en un sistema de asociaciones (Stiles et al., 1990). La asimilación representa que cuando aparece una experiencia problemática el sí mismo logra aceptarla. La asimilación es el resulta$d o$, la consecuencia de un proceso. La APES presenta una secuencia sobre la relación cambiante entre las experiencias problemáticas y las voces dominantes. Las voces dominantes y no dominantes deben cambiar en el proceso de construir un puente de significado, es decir, una comprensión entre ambas, para que se produzca un progreso en la asimilación.

El concepto clave para comprender el modelo de asimilación y por qué se produce la asimilación de experiencias es, pues, el concepto de puente de significado. El puente de significado se refiere a un signo o a un sistema de signos que tiene el mismo significado tanto para el autor, como para quien va dirigido. En el insight psicoterapéutico, el puente de significado se da entre las voces internas y consta de signos que tienen el mismo significado para la voz problemática, así como para la comunidad dominante de voces (Stiles \& Glick Brinegar, 2007, p. 103). El puente de significado tiene, pues, lugar, fundamentalmente en el nivel 4 o de Insight y representa el esquema que vincula las voces (Honos-Webb \& Stiles, 1998). Por ejemplo, una paciente, María, analizada con la APES hizo un puente de significado que le permitió minimizar la importancia de sus 
voces dominantes ("María como persona que controla y resuelve las cosas"), asumiendo que había experiencias problemáticas que no podía controlar y con las que tenía que aprender a convivir (voces no dominantes: mareo, cansacio y agotamiento), haciendo un nuevo esquema sobre ella misma. Así María reconoció que era una persona que, aunque con problemas, podía tener un funcionamiento adecuado, aceptando sus experiencias negativas que inicialmente rechazaba.

\section{Procedimiento a seguir para el estudio con la APES}

La APES puede aplicarse a cualquier muestra de material escrito $\mathrm{u}$ oral, aunque fundamentalmente se ha aplicado a transcripciones de terapia y, recientemente, como expondremos más adelante a narraciones de pacientes. Para preparar los datos se siguen las normas y recomendaciones establecidas por el grupo de Stiles (Stiles et al. 1990; Stiles, et al., 1992; Honos-Webb, et al., 1999; Knobloch, Endres, Stiles, \& Silberschatz, 2001; Lani et al., 2002).

Básicamente se hace un catálogo de temas y voces principales (dominantes y no dominantes) que se identifican mediante un conocimiento exhaustivo del caso, de las transcripciones y mediante el seguimiento de una serie de criterios. Así, según Osatuke et al. (2004), las voces pueden identificarse a partir de determinadas características que se expresan de manera consistente en lo dicho por los pacientes. De manera más concreta, las voces pueden reconocerse a partir de su intencionalidad (lo que una persona intenta transmitir desde la perspectiva de una voz en concreto), su función (aquello que consigue la persona con su verbalización: expresar sentimientos, sacar conclusiones, etc.), su contenido (personas, acontecimientos, emociones, etc. acerca de los cuales se habla), su afecto (tipo de emoción expresada), sus disparadores contextuales (elementos internos o externos que activan aparentemente la expresión de una voz determinada) o por su patrón dialogal (patrones en los que se repiten determinadas secuencias o configuraciones de las voces y que sólo pueden ser entendidas en el contexto de la sesión).

Cuando se tiene preparado el catálogo de temas y voces se pasa a aplicar la APES siguiendo las normas previstas en los manuales. En esta parte del procedimiento, una cuestión importante es el empleo de jueces. Aunque los datos cualitativos dependen mucho del contexto y del conocimiento del caso, es aconsejable buscar un acuerdo entre jueces. Con ello se garantiza la validez de los datos alcanzados. En palabras de Stiles (2007), el consenso entre los observadores múltiples puede ayudar a hacer el producto más convincente a los otros y éste es un punto fundamental en los estudios cualitativos. Como recogen Schielke, Fishman, Osatuke, \& Stiles (2009) las conclusiones sugieren que los grupos que toman decisiones por consenso trabajan y responden igual o mejor que cada individuo de manera individual.

Una vez hecha esta exposición del modelo de asimilación pasaremos a resumir, como punto final, algunos de los principales resultados alcanzados con la APES, en nuestro contexto.

\section{Principales resultados en diversos estudios sobre el proceso de asimilación}

La mayoría de los estudios realizados se han hecho en el marco de la terapia lingüística de evaluación (Caro Gabalda, 1994), como ya se ha dicho. Los primeros estudios se hicieron con acontecimientos terapéuticos, dentro del paradigma de procesos señalado por Rice \& Greenberg (1984). En este sentido, analizando acontecimientos significativos de cambio se encontró que la resolución de experiencias problemáticas se relacionaba con asimilación, mientras que la no resolución se relacionaba con dificultades y problemas en la asimilación (Caro Gabalda, 2003a,b).

Tras estos primeros estudios se pasó a analizar procesos completos de terapia. Es decir, se grabaron y transcribieron todas las sesiones de terapia y se analizaron con la APES. En este sentido se ha encontrado que la asimilación se relaciona con cambio terapéutico (Caro Gabalda, 2006a, 2007, 2008, en prensa), mientras que la no asimilación de experiencias problemáticas se manifiesta en pacientes con escaso éxito terapéutico (Caro Gabalda, 2006b, en prensa). Todo ello en comparaciones estándar pre-test-post-test. De esta manera reproducimos, en 
nuestro contexto terapéutico, los mismos datos ya encontrados por otros investigadores con la APES (véase una revisión de ellos en Stiles, 2002; Detert et al., 2006; Goodridge \& Hardy, 2009), así como nuestros primeros estudios arriba mencionados.

Uno de los resultados más constantes ha sido el tipo de patrón de asimilación. Es decir, si originalmente, como ya se ha señalado, el patrón de asimilación se suponía fluido y sin grandes retrocesos entre los niveles APES, pronto se vio que esto no era así (Stiles, et al., 1990, 1991). Este resultado es especialmente relevante o característico de los estudios con la APES en contextos de terapias cognitivocomportamentales (Goodridge \& Hardy, 2009; Osatuke, et al., 2005; Stiles, 2005). Este patrón dentado, es decir, con avances y retrocesos en los niveles APES es el característico en los estudios en el marco de la TLE. Esto es, con independencia del éxito o fracaso en la terapia, lo que hemos encontrado son numerosos retrocesos (Caro Gabalda \& Stiles, 2009). Esto nos lleva a asumir que las distintas psicoterapias presentan patrones de asimilación diferentes. Esta cuestión de los retrocesos es uno de los aspectos que estamos estudiando en la actualidad: cómo y por qué se producen los retrocesos, y por qué aparecen en pacientes con, y sin éxito, terapéutico. En la figura 1 tenemos un ejemplo de este proceso dentado de asimilación tomado de María (Caro Gabalda, 2007) una paciente tratada con la TLE y analizada con la APES.

Con el tiempo hemos ido abriendo otras líneas de investigación en la línea de los estudios de caso que comprueban una teoría (Stiles, 2003). Así, hemos estudiado la asimilación de experiencias problemáticas en adolescentes, así como la asimilación de experiencias problemáticas a través de narraciones y en contextos diferentes a los tradicionalmente empleados en el modelo de asimilación. Resumiremos, brevemente, algunos de estos estudios.

En cuanto a nuestro estudio con adolescentes (Rojo Serrano, 2011) hemos encontrado una situación semejante a la encontrada en el contexto de la TLE. Es decir, una adolescente diagnosticada con depresión, y tratada desde una perspectiva cognitivo-comportamental, presentaba diversas experiencias problemáticas, que fue asimilando a lo largo del tratamiento.

El tratamiento tuvo lugar con éxito (en la comparación pre-test-post-test) a lo largo de 14 sesiones. De manera, que podemos relacionar cambio con asimilación en este primer estudio con adolescentes. El patrón que mostró también fue dentado, con retrocesos entre los niveles APES, al igual que en los casos con adultos analizados previamente.

Figura 1. Proceso de asimilación de la experiencia problemática de incapacidad para hacer cosas

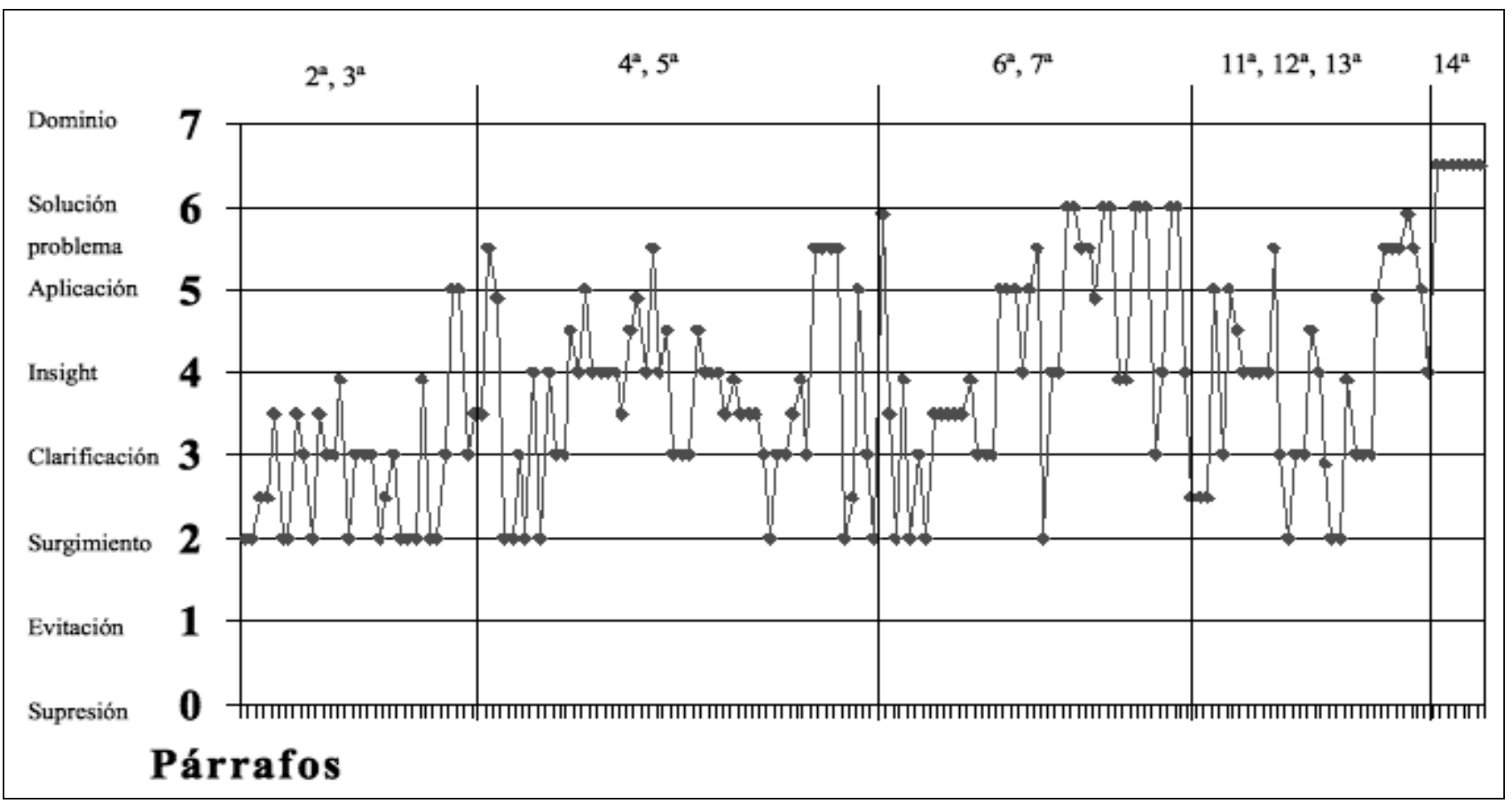


Los estudios con narraciones se han hecho en dos campos. En primer lugar, cabe reseñar un estudio de caso (Allepuz Faus, 2010) con una participante de 24 años que presentaba un historial oncológico: fibroadenomas en ambas mamas y quistes ováricos y que tuvo que enfrentarse a estas experiencias problemáticas. La participante escribió a lo largo de 9 meses 34 narraciones, a raíz de 1 por semana en las que escribía libremente sobre su experiencia oncológica. Los datos muestran asimilación de algunas experiencias problemáticas, así como una disminución de sus puntuaciones pretest-postest. Igualmente, el patrón que se presenta es dentado. Es decir, que a pesar de no haber interacción pacienteterapeuta, ya que la participante se enfrentó ella sola a sus experiencias problemáticas, mediante sus narraciones, los procesos de asimilación son igualmente dentados.

El último estudio que nos gustaría reseñar se centra en las narraciones de 4 participantes, familiares de enfermos fallecidos de Esclerosis Lateral Amiotrófica (ELA) (Yera Sabater, 2011). Los participantes fueron invitados a escribir durante 10 sesiones sobre la experiencia problemática de la muerte por ELA del familiar. Al mismo tiempo recibieron sesiones de counseling psicológico. Aquí nos encontramos con una situación diferente a las anteriores, ya que estos participantes no asimilaron todas sus experiencias relacionadas con el duelo, mostrando oscilaciones entre niveles altos, interme- dios y bajos de asimilación. Aunque sí disminuyeron sus puntuaciones en las pruebas pasadas en el postest. Estos datos sugieren que no todas las experiencias son igualmente asimilables (véase al respecto Varvin \& Stiles, 1999). Así la ELA es una enfermedad difícil para el enfermo y sus familiares que puede necesitar más tiempo para asimilarse. Teniendo en cuenta que los participantes escribieron sus narraciones sólo a lo largo de 10 sesiones queda como una cuestión abierta estudiar el proceso de asimilación con pacientes en duelo tras una enfermedad degenerativa de este tipo. Aún así, la mayoría de los procesos de asimilación fueron, al igual que lo encontrado en los estudios anteriores, de tipo dentado.

\section{Conclusión}

Como vemos, el modelo de asimilación se está desarrollando en diversos contextos, lo que lo mantiene en evolución y como un modelo adecuado para describir el proceso del cambio. Su estilo integrador nos permite emplearlo en cualquier contexto, terapéutico o no, así como con diversos tipos de participantes. Es un modelo abierto, sustentado por los diversos estudios, que contribuye a encontrar elementos importantes para entender cómo los seres humanos asimilamos experiencias problemáticas.

\section{Extended Summary}

Process research has as a main aim to describe, explain and predict therapeutic changes in contrast to outcome research, which aims to show the effectiveness of the therapeutic methods. In the process research context, we focus on the model of assimilation of problematic experiences developed by Stiles and his group. According to the assimilation model in a successful therapy, clients follow a regular sequence when they are assimilating painful or problematic experiences. That is, the model describes the process whereby clients are able to integrate into their self the painful or problematic experiences they are facing.

The assimilation model is an integrative, concise and coherent one that identifies common change processes and articulates systematic differences between therapeutic approaches. It has been applied in a variety of therapeutic and other contexts, with a variety of patients.

The assimilation model it is not a prescriptive model; that is, it does not recommend that clinicians follow a particular therapeutic procedure or specify what they should do for producing the changes. Consequently the model can be used in different therapeutic settings. From a theoretical point of view the model has drawn on Piaget's thinking about schemata, from Rogers' thinking about the self development and on Elliott's thinking about helpful events during psychotherapy.

Theoretically, an experience is considered prob- 
lematic because it is emotionally disequilibrating. Such experiences often appear in response to a great range of events (usually of an interpersonal kind), but they are incompatible with our usual way of thinking and acting and they cannot be successfully represented in consciousness. Instead, the problematic experiences are denied, distorted or are erroneously represented, producing dysforic feelings and maladaptive behaviour.

In this context, the model differentiates between dominant and nondominant voices. Dominant voices are our resources, our usual way of thinking, feeling and behaving. Nondominant voices are our problematic experiences incompatible with our dominant voices.

From these studies and from a series of intensive case studies Stiles and his group have developed the Assimilation of Problematic Experiences Scale or $A P E S$, which has eight stages or levels that are postulated as a continuum, from warding off the problematic experience until its mastery. The stages are:

0: Warded off: Content is unformed; client is unaware of the problem. Distress may be minimal, reflecting successful avoidance

1: Unwanted thoughts: Content is distressing thoughts. Client prefers not to think about it; topics are raised by therapist or external circumstances. Affect is often more salient than the content and involves strong negative feelings -anxiety, fear, anger, sadness.

2: Vague awareness/emergence: Client acknowledges the problematic experience and describes distressing associated thoughts but cannot formulate the problem clearly. Affect includes acute psychological pain or panic associated with the problematic thoughts and experiences.

3: Problem statement/clarification: Content includes a clear statement of a problem -something that could be worked on. Affect is negative but manageable, not panicky.

4: Insight: The problematic experience is placed into a schema, formulated, understood, with clear connective links. Affect may be mixed, with some unpleasant recognitions, but also with curiosity or even pleasant surprise.

5: Application: The understanding is used to work on a problem; there are specific problem-solving efforts. Clients may describe considering alternatives or systematically selecting courses of action. Affective tone is positive, businesslike, optimistic.

6: Problem solution: Client achieves a solution for a specific problem. Affect is positive, satisfied, proud of accomplishment. As the problem recedes, affect becomes more neutral.

7: Mastery: Client successfully uses solutions in new situations; this generalizing is largely automatic, not salient. Affect is neutral (i.e., this is no longer something to get excited about).

Passages analyzed with APES may be rated at intermediate stages: for instance a rating of 2.5 conveys the meaning that the patient is right between vague awareness and clarification.

From a process research perspective, APES studies are included more specifically in the events paradigm which studies brief therapeutic episodes sharing a common marker. APES studies follow this paradigm while examining selected problems and how they are worked on time after time during sessions.

The APES classifies therapeutic patterns (in a minor, microanalytic scale) which are intensively analyzed and in a given context. Although APES could be investigated from a quantitative perspective most of the research has been done from a qualitative stance.

We would like to emphasize some of the main results and assumptions with the assimilation model:

- Clients may enter therapy or introduce a problem at any APES stage, although obviously it would be unusual to find stages 6 (Problem solution) and 7 (Mastery) at the beginning of the therapy.

- The different psychotherapies appear to prefer to focus at different ranges along the APES continuum and put in practice different strategies in relation to the clients' degree of assimilation of their experiences. Experiential and psychodynamic therapies seem to focus on unassimilated experiences and work toward its understanding, whereas cognitive and behavioral therapies focus on known problems and work toward the application of rational solutions to practical situations. However, this is not a clear-cut distinction. 
- The content of the elaborated schemas may be different depending on the kind of therapy. For instance, cognitive therapies focus on the identification of self-defeating, irrational thoughts and on their underlying dysfunctional attitudes. Theoretical labels (for instance, cognitive distortions) are applied to this thought patterns and tasks are prescribed for correcting them.

However, from this background a major conceptual and methodological puzzle has arisen. It concerns the fundamental hypothesis that problems progress through a systematic sequence when they are being assimilated and to the possibility of its maintenance.

- The hypothesis that there is a regular sequence of change, has not been confirmed in detail. Although a problem's progress in therapy generally tends to move from one stage to another one of the APES, this progress shows many moments where there are setbacks, or forward leaps between the stages. This deviation from the expected pattern has appeared more clearly in directive therapies, such as cognitive-behavioral therapy and in the linguistic therapy of evaluation than in non-directive therapies.

Several hypotheses have previously been offered for explaining these setbacks. One hypothesis involves the concept of the therapeutic zone of proximal development that Stiles developed with Leiman. Other hypotheses suggest that there are multiple strands of a problem, interference with life events or with other client's problematic experiences, multiple internal perspectives. From the linguistic therapy of evaluation we have assumed as an explicative hypothesis the balance metaphor.

Assimilation studies work mainly with transcripts and have analyzed therapeutic sessions in a different range of therapeutic models. However, other studies could be done using narratives.

For preparing the transcriptions in order to analyze assimilation processes our group has followed the guidelines devised by Stiles' group and which have been published in several papers. The guidelines for preparing samples to be analyzed are:

1. Cataloguing: labeling each topic, defined as the client's expressed attitude toward an object, with the aim of having a catalogue of thoughts units, that is, one or more utterances that are judged to go together. For instance, a client within her speaking turn says in relation to her dizziness: "Really, I feel bad when I realize I am dizzy; and I need to understand how and why I am feeling this way". In this speaking turn there would be two thoughts units, the first one is referred to the information the client is given about feeling unwell ("really, I feel bad when I realize I am dizzy"), the second would be composed of the client's utterance where she manifests her attitude of understanding how and why is she feeling unwell ("and I need to understand how and why I am feeling this way").

2. Finding insights: finding a cognitive assimilation of a problematic experience to an active schema. This strategy insured that the problematic experiences would be ones that underwent some change during treatment

3. Excerpting: searching through the catalogue of topics seeking related thoughts units. The aim is to have a catalogue (with the topics, situations and problematic experiences shown in different occasions) which allows us to select several moments where clients attitudes about several problematic experiences are shown.

4. Applying the APES to the selected thoughts units, following the group's manuals from 1998 and 2002. The manuals include markers, that is, "clues" about each APES stage that Stiles' group has been improving during their studies.

5. Using raters: although qualitative data are highly dependent on context and of the clinical case knowledge, it is possible to look for raters agreement. In this sense the members of the research group discuss and agree about each one of the codes following the present qualitative studies approaches followed by Stiles' group.

Finally, our paper makes a review about the main studies found in two contexts: with clients treated with linguistic therapy of evaluation or an adolescent treated with a cognitive-behavioral perspective and with clients that do not receive therapy, but used narratives to cope with their experiences. From a gener- 
al perspective we could relate change with progress in assimilation, no change with difficulties in assimilation in analyses of whole processes of therapy (with transcripts of sessions) and with narratives.

\section{Referencias}

Allepuz Faus, A. (2010). La asimilación de experiencias problemáticas a través de narraciones: Un estudio de caso. Trabajo final de Máster. Universidad de Valencia.

Brinegar, M. G., Salvi, L. M., Stiles, W. B., \& Greenberg, L.S. (2006). Building a meaning bridge. Therapeutic progress from problem formulation to understanding. Journal of Counseling Psychology, 53, 165-180.

Caro Gabalda, I. (1994). La práctica de la terapia lingüística de evaluación. Salamanca: Amarú Ediciones.

Caro Gabalda, I. (2003a). La resolución de experiencias problemáticas en la terapia lingüística de evaluación según el modelo de asimilación (I). Boletín de Psicología, 79, 31-57.

Caro Gabalda, I. (2003b). La resolución de experiencias problemáticas en la terapia lingüística de evaluación según el modelo de asimilación: El análisis de Silvia (II). Boletín de Psicología, 79, 59-84.

Caro Gabalda, I. (2006a). The assimilation of problematic experiences in linguistic therapy of evaluation: How did María assimilate the experience of dizziness? Psychotherapy Research, 16, 422435.

Caro Gabalda, I. (2006b). The assimilation of problematic experiences in the context of a therapeutic failure. Psychotherapy Research, 16, 436-452.

Caro Gabalda, I. (2007). La asimilación de experiencias problemáticas en la terapia lingüística de evaluación: ¿Cómo asimiló María para hacer cosas? Boletín de Psicología, 89, 47-73.

Caro Gabalda, I. (2008). Assimilation of problematic experiences: A case study. Journal of Constructivist Psychology, 21, 151-172.

Caro Gabalda, I., \& Stiles, W. B. (2009). Retrocesos no contexto de terapia linguística de avaliaçâo. Análise Psicologica, 2 (XXVII), 199-212.
Caro Gabalda, I. (en prensa). El cambio terapéutico a través del modelo de asimilación: Su aplicación en la terapia lingüística de evaluación. Revista de Psicopatología y Psicología Clínica.

Castonguay, L. G., \& Beutler, L. E. (2006). Principles of therapeutic change: A Task Force on participants, relationships, and techniques factors. Journal of Clinical Psychology, 62, 631638.

Detert, N. B., Llewelyn, S., Hardy, G. E., Barkham, M., \& Stiles, W. B. (2006). Assimilation in goodand poor-outcome cases of very brief psychotherapy for mild depression: An initial comparison. Psychotherapy Research, 16, 393-407.

Field, S. D., Barkham, M., Shapiro, D. A., \& Stiles, W.B. (1994). Assessment of assimilation in psychotherapy: A quantitative case study of problematic experiences with a significant other. Journal of Counseling Psychology, 41, 397-406.

Goldsmith, J. Z., Mosher, J. K., Stiles, W. B., \& Greenberg, L. S. (2008). Speaking with the client's voices: How a person-centered therapist used reflections to facilitate assimilation. PersonCentered and Experiential Psychotherapies, 7, 3, 155-172.

Goodridge, D. \& Hardy, G. E. (2009). Patterns of change in psychotherapy: An investigation of sudden gains in cognitive therapy using the assimilation model. Psychotherapy Research, 19, 114-123.

Henry, H., Stiles, W. B., \& Biran, M. W. (2005). Loss and mourning in immigration: Using the assimilation model to assess continuing bonds with native culture. Counselling Psychology Quarterly, 18, 109-120.

Hermans, H. J. M. (1996). Voicing the self: From information processing to dialogical interchange. Psychological Bulletin, 119, 31-50.

Hermans, H. J. M., Kempen, H. J. G., \& van Loon, R. P. P. (1992). The dialogical self: Beyond individualism and rationalism. American Psychologist, 47, 23-33.

Honos-Webb, L. \& Stiles, W.B. (1998). Reformulation of assimilation analysis in terms of voices. Psychotherapy, 35, 23-33.

Honos-Webb, L., Surko, M., Stiles, W. B., \& Greenberg, L. S. (1999). Assimilation of voices in 
psychotherapy: The case of Jan. Journal of Counseling Psychology, 46, 448-460.

Humphreys, C. L., Rubin, J. S., Knudson, R. M., \& Stiles, W. B (2005). The assimilation of anger in a case of dissociative identity disorder. Counselling Psychology Quarterly, 18, 121-132.

Knobloch, L. M., Endres, L. M., Stiles, W. B., \& Silberschatz, G. (2001). Convergence and divergence of themes in successful psychotherapy: An assimilation analysis. Psychotherapy, 38, 31-39.

Lani, J. A., Glick, M. J., Osatuke, K., Brandenburg, C., Gray, M. A., Humphreys, C. M., Reynolds, D., \& Stiles, W. B. (2002). Markers of assimilation manual. Manuscrito sin publicar. Universidad de Ohio.

Norcross, J. N. (2002) (Ed.). Psychotherapy relationships that work. Nueva York: Oxford University Press.

Osatuke, K., Glick, M. J., Gray, M. A., Reynolds, D., Humphreys, C. L., Salvi, L. M., \& Stiles, W. B. (2004). Assimilation and narrative. Stories as meaning bridges. En L. Angus \& J. McLeod (Eds.), The handbook of narrative and psychotherapy. Thousand Oaks: Sage.

Osatuke, K., Glick, M. J., Stiles, W. B., Greenberg, L. S., Shapiro, D. A., \& Barkham, M. (2005). Temporal patterns of improvement in clientcentered therapy and cognitive-behaviour therapy. Counselling Psychology Quarterly, 18, 95108.

Osatuke, K. \& Stiles, W.B. (2006). Problematic internal voices in clients with borderline features: An elaboration of the assimilation model. Journal of Constructivist Psychology, 19, 287-319.

Osatuke, K. \& Stiles, W. B. (en prensa). Change in postraumatic stress disorder: An assimilation model account. En G. Dimaggio \& P.H. Lysaker (Eds.), Metacognition and severe adult mental disorders: From basic research to treatment. Londres: Routledge.

Reid, M. \& Osatuke, K. (2006). Acknowledging problematic voices: Processes occuring at early stages of conflict assimilation in patients with functional somatic disorder. Psychology and Psychotherapy, 79, 539-555.

Rice, L. \& Greenberg, L. (1984). The new research paradigm. En L. Rice \& L. Greenberg (Eds.),
Patterns of change (pp. 7-26). Nueva York: Guilford Press.

Rojo Serrano, I. (2011). El caso de Bárbara: La asimilación de experiencias problemáticas en una adolescente. Trabajo final de Máster. Universidad de Valencia.

Schielke, H. J., Fishman, J. L., Osatuke, K., \& Stiles, W. B. (2009). Creative consensus on interpretations of qualitative data: The Ward Method. Psy-chotherapy Research, 19, 1-8.

Stiles, W. B. (2002). Assimilation of problematic experiences. En J.C. Norcross (Ed.), Psychotherapy relationships that works (pp. 357-365). Cary: Oxford University Press

Stiles, W. B. (2003). When is a case study scientific research? Psychotherapy Bulletin, 38, 6-11.

Stiles, W. B. (2005). Extending the Assimilation of Problematic Experiencies Scale: Commentary on the special issue. Counselling Psychology Quarterly, 18, 85-94.

Stiles, W. B. (2006). Assimilation and the process of outcome: Introduction to a special section. Psychotherapy Research, 16, 389-392.

Stiles, W. B. (2007). Theory-building case studies of counselling and psychotherapy. Counselling and Psychotherapy Research, 7, 122-127.

Stiles, W. B., Barkham, M., Shapiro, D. A., \& FirthCozens, J. (1992). Treatment order and thematic continuity between contrasting psychotherapies: Exploring an implication of the assimilation model. Psychotherapy Research, 2, 112-124.

Stiles, W. B., Elliott, R., Llewelyn, S., Firth-Cozens, J., Margison, F. R., Shapiro, D. A., \& Hardy, G. (1990). Assimilation of problematic experiences by clients in psychotherapy. Psychotherapy, 27, 411-420.

Stiles, W. B. \& Glick Brinegar, M. (2007). Insight as a stage of assimilation: A theoretical perspective. En L .G. Castonguay \& C. E. Hill (Eds.), Insight in psychotherapy (pp. 101-118). Washington: APA.

Stiles, W. B., Honos-Webb, L., \& Lani, J. A. (1999). Some functions of narrative in the assimilation of problematic experiences. Journal of Clinical Psychology, 55, 1213-1226.

Stiles, W. B., Meshot, C. M. Anderson, T. M., \& Sloan, W. W. (1992). Assimilation of problematic 
experiences: The case of John Jones. Psychotherapy Research, 2, 81-101.

Stiles, W. B., Morrison, L. A., \& Haw, S. (1987). Manual for assessing assimilation of problematic experiences in psychotherapy sessions. Manuscrito sin publicar. MRC/ESRC SAPU.

Stiles, W. B., Morrison, L. A., Haw, S. K., Harper, H., Shapiro, D. A., \& Firth-Cozens, J., (1991). Longitudinal study of assimilation in exploratory psychotherapy. Psychotherapy, 28, 105-206.

Stiles, W. B. \& Shapiro, D. A. (1989). Abuse of the drug metaphor in psychotherapy process-outcome research. Clinical Psychology Review, 9, 521-543.

Stiles, W. B. \& Shapiro, D. A. (1994). Disabuse of the drug metaphor: Psychotherapy and process- outcome correlations. Journal of Consulting and Clinical Psychology, 62, 942-948.

Stiles, W. B., Shapiro, D.A., Harper, H., \& Morrison, L. A. (1995). Therapist contributions to psychotherapeutic assimilation: An alternative to the drug metaphor. British Journal of Medical Psychology, 68, 1-13.

Varvin, S. \& Stiles, W. B. (1999). Emergence of severe traumatic experiences: An assimilation analysis of psychoanalytic therapy with a political refugee. Psychotherapy Research, 9, 381-404.

Yera Sabater, A. (2011). Asimilación del duelo en familiares de enfermos de esclerosis lateral amiotrófica (ELA). Trabajo final de Máster. Universidad de Valencia.

Artículo recibido: 09/06/2011 Revisión recibida:14/07/2011

Aceptado: 14/09/2011 